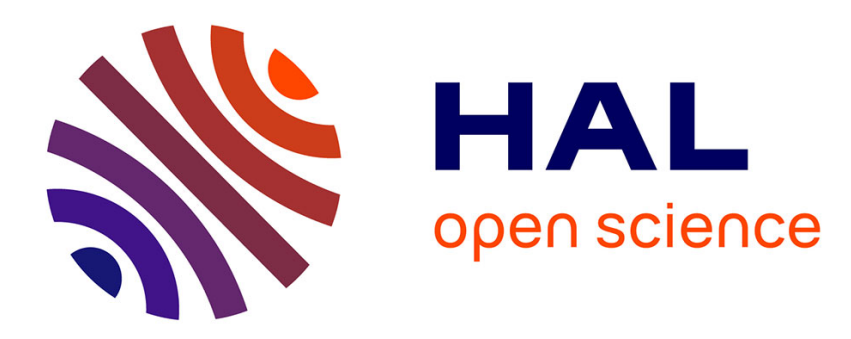

\title{
La psychothérapie des patients psychotiques hallucinés Guy Gimenez
}

\section{To cite this version:}

Guy Gimenez. La psychothérapie des patients psychotiques hallucinés. Cahiers de psychologie clinique , 2003, 2 (21), pp. 83 - 98. 10.3917/cpc.021.0083 . hal-01384745

\section{HAL Id: hal-01384745 https://hal-amu.archives-ouvertes.fr/hal-01384745}

Submitted on 24 Oct 2016

HAL is a multi-disciplinary open access archive for the deposit and dissemination of scientific research documents, whether they are published or not. The documents may come from teaching and research institutions in France or abroad, or from public or private research centers.
L'archive ouverte pluridisciplinaire HAL, est destinée au dépôt et à la diffusion de documents scientifiques de niveau recherche, publiés ou non, émanant des établissements d'enseignement et de recherche français ou étrangers, des laboratoires publics ou privés. 


\section{LA PSYCHOTHÉRAPIE DES PATIENTS PSYCHOTIQUES HALLUCINÉS}

\section{Guy GIMENEZ*}

\section{Introduction}

Le suivi psychothérapique de patients psychotiques en crise et régresses, délirants et souvent hallucinés, m'a amené à me poser des questions pragmatiques, concernant la prise en compte des hallucinations dans les thérapies d'inspirations psychanalytiques. Comment travailler avec des patients qui hallucinent pendant les entretiens ? Estil pertinent de prendre en compte ces hallucinations ? Comment alors intervenir ? Y-a-t-il alors un risque, comme on l'entend souvent, d'entrer dans le délire du patient ? Ces questions m'ont amené à contribuer à la construction d'un modèle de l'hallucination. Celui-ci prend appui sur les grandes hypothèses de Freud et de ses successeurs, concernant la psychose.

\section{Les deux temps de la psychose}

Ce modèle considère que l'hallucination est le résultat d'un conflit entre le Moi et un fragment de la réalité externe intolérable qui confronte le sujet à une expérience (sensori-affective) dont le modèle pourrait être le trauma affectif. Ce conflit (primaire) est géré en deux temps (Freud 1924a \& b). Le premier temps vise à se défendre contre un fragment de réalité intolérable, le second temps, lui, est une
* Psychologue, Maître de Conférences en Psychologie Clinique et Psychopathologie, Université de Provence, Aix-Marseille, l'Orée du village 6,13880 Velaux, France. 
1 Dans son œuvre, S. Freud utilisera différents termes pour traduire ce qui, du conflit psychique, a été gommé, effacé, repoussé, rejeté, aboli. Nous utiliserons le terme de rejet comme un terme générique rendant compte des différents termes utilisés par Freud pour ce mécanisme défensif. 2 Mais cette traduction du terme allemand Verwerfung par forclusion ne se fait de façon stable qu'à partir de 1955-56. J. Lacan parle de rejet ou refus dans son Séminaire I sur les Ecrits techniques de Freud (1953-54, 54). Lacan propose dans son œuvre de traduire Verwerfung par différents termes : retranchement (1954, 386), expulsion, refus, rejet (1955-56, 61), puis « forclusion » qu'il adoptera (1955-56). II s'agit d'un terme qui « dans son acception originelle juridique, signifie la déchéance d'un droit non exercé dans les délais prescrits » (Nasio, 1987, 226, note 13). Avec Laplanche et Pontalis (1967), nous pensons, de plus, que d'un « point de vue terminologique, l'usage du terme Verwerfung ne recouvre pas toujours ce que connote «forclusion » et, à l'inverse, que d'autres termes freudiens désignent ce que $\mathrm{J}$. Lacan cherche à mettre en évidence $»$ reconstruction qui consiste à remplacer le fragment de réalité insupportable par une nouvelle réalité : la néo-réalité hallucinatoire (Freud, 1924 a, 285). Le premier temps, défensif (ou « négatif ») est soustendu par les mécanismes de déni et de rejet. Dans ce premier temps, le mouvement défensif porte d'une part sur la réalité externe intolérable (c'est le déni) et sur la « représentation potentielle » que le sujet aurait pu en avoir (c'est le rejet, ou la forclusion locale).

\section{$I^{\circ}$ Le déni et le rejet}

IIy a dans ce premier temps déni du fragment de réalité intolérable. Le déni (Verleugnung) est un mécanisme de défense qui porte sur une partie de la réalité externe et tout se passe comme si ce fragment de réalité (perception, événement) n'avait jamais existé. Il y a en même temps, rejet de la représentation que le patient aurait pu avoir de cette réalité.

Le rejet' (Verwerfung) est un mécanisme de défense beaucoup plus puissant que le refoulement et qui porte sur une représentation intolérable et sur l'affect qui lui est conjoint ; tout se passe, dit Freud (1894, 11-12), comme si la représentation n'était pas parvenue jusqu'au moi. Le rejet de la représentation deviendra chez Lacan la « forclusion du signifiant »(la forclusion locale $)^{2}$. Le rejet ne porte pas sur une représentation déjà construite (comme c'est le cas pour le refoulement), mais sur une représentation en construction qui risque de créer un conflit intolérable. Le rejet « tue dans l'œuf » la construction de la représentation $^{3}$. Elle en attaque le processus élaboratif : nous sommes ici proches de l'attaque contre la liaison décrite par Bion. Le rejet produit la destruction des « connections avec les représentations de mots » dont parle Freud (1915). Il s'agit d'un mécanisme d'antisymbolisation.

L'expérience s'inscrit ainsi sous forme de traces mnésiques brutes, non symbolisées, non mentalisée : c'est-à-dire sous forme de représentations de choses.

Je propose d'appeler « représentation potentielle » la représentation ou la pensée qui n'a pu se construire entièrement à cause du mécanisme de rejet. La « représentation potentielle »(Gimenez, 1994) est la représentation de la situation intolérable, inassimilable, dont la constitution ne serait pas parvenue à son terme (à cause du mécanisme de rejet), mais qui a été attaquée, détruite, rejetée, « tuée dans l'œuf » avant même de se constituer ${ }^{4}$. Nous avons ici une différence radicale avec le refoulement, qui, lui, porte sur une représentation constituée. 
Freud appelle « représentation inconciliable » ou « représentation insupportable » la représentation sur laquelle porte le rejet (Freud, 1894, 12-13). Je soutiens l'hypothèse que c'est à partir de cette représentation potentielle que va se construire l'hallucination.

\section{$2^{\circ}$ Déni et rejet : deux faces d'un même processus}

Ainsi, ce premier temps défensif, est-il sous-tendu par le déni d'un fragment de réalité intolérable et par le rejet de la représentation que le patient aurait pu en avoir. Je soutiens que les mécanismes de rejet et de déni constituent deux faces d'un même processus. Ils constituent le premier temps défensif de l'hallucination. Dans cette perspective, le rejet porterait en même temps sur le processus même de symbolisation de la situation intolérable (traumatique), et sur la représentation potentielle que le sujet pourrait en avoir ${ }^{5}$. La réalité externe serait alors appréhendée par la psyché sans que soit porté sur elle le jugement de son existence (Bejahung) ${ }^{6}$.

\section{$3^{\circ}$ Le temps négatif et les hallucinations négatives}

Ce premier temps de l'hallucination (déni, rejet) confronte le sujet à un vide, une faille, une béance. Un rien intolérable, du « négatif ». Ce mouvement se repère bien dans les hallucinations négatives : je regarde un objet mais tout se passe comme si je ne le percevais pas. Dans cette perspective, ce premier temps négatif est sous-jacent à toute hallucination psychotique, elle en constitue la toile de fond. L'hallucination négative correspond à ce premier temps, quand il n' est pas suivi de reconstruction (Gimenez, 1994). Elle est pour moi un déni perceptif : l'hallucination négative est à la perception ce que le déni est à la pensée. Elle est une « présentation » d'absence de représentation symbolisée (Green, 1977 ; 1993, 262) ${ }^{7}$, hallucination de fond gommant le découpage d'une forme. Ce déni perceptif fonctionne comme la suppression active d'une perception insupportable. Je soutiens que le mouvement négatif de l'hallucination porte d'une part sur un fragment de réalité du monde externe (la situation intolérable) qui a provoqué le conflit psychique (Freud, 1924 b, 300), et d'autre part sur la représentation que le sujet pourrait en avoir (Gimenez, 1994,1997, 2000b). (suite note 2) (Laplanche, Pontalis, 1967, 165). II s'agit par exemple des termes : ablehnen (écarter, •décliner), aufheben (supprimer, abolir), ver/eugnen (dénier) (Laplanche, Pontalis, 1967, 164).

3 Comme le dit J.D. Nasio « L'opération forclusive n'agit pas sur un élément mais tue dans l'œuf un mouvement attendu. Le forclos c'est du non-arrivé plutôt que du rejeté ; et la forclusion, une impuissance à exister plutôt qu'un rejet » (Nasio, 1987, 121). Le rejet arrête la représentation dans sa construction ou détruit une représentation déjà construite. En d'autres termes, le rejet porte sur la construction même de la représentation ou de la pensée.

4 II s'agit là du travail du négatif, attaque de la pensée, envie, destructivité, pulsion de mort, autant d'appréhension d'un même processus.

5 Représentation que Freud (1894) nomme la « représentation inconciliable », la « représentation menacée », la « représentation insupportable».

6 Freud dit à propos de L'homme aux loups : « Nous savons déjà quelle attitude notre patient avait d'abord adoptée en face du problème de la castration. II la rejeta et 


\section{Les reconstructions délirante et hallucinatoire}

\section{$1^{\circ}$ Reconstruction}

Très souvent ce premier temps, défensif, est suivi d'un second. Celui de la reconstruction d'une néo-réalité hallucinatoire qui permet au sujet de ne pas se confronter au vide du premier temps défensif. Cette création est une tentative de guérison (Freud, 1924a), de cicatrisation (Rosolato, 1964), de réparation (Freud, 1924a \& b) $)^{8}$.

(suite note 6) s'en tint à la théorie du commerce par l'anus. Quand je dis : il la rejeta, le sens immédiat de cette expression est qu'il n'en voulut rien savoir au sens du refoulement. Aucun jugement n'était là porté sur la question de son existence, mais les choses se passaient comme si elles n'existaient pas » (Freud, 1914c,389).

7 L'expression de A. Green est « représentation d'absence de représentation » $(1977$, 1993, 262).

8 Pour Freud, I'hallucination est une tentative de guérison et de réparation en ce qu'elle vise à re construire une certaine continuité avec la réalité externe.

9 Freud nous le rappelle en 1924 : « Pour la névrose comme pour la psychose, la question qui vient à se poser n'est pas seulement celle de la perte de la réalité, mais aussi celle d'un substitut de la réalité» (Freud, 1924b, 303).
Le sujet n'est ainsi pas confronté au « négatif », au vide laissé par le premier temps. La nouvelle réalité recouvre le « négatif » ce qui nous fait quelquefois oublier que le centre de la psychose c'est ce premier temps destructif et non la reconstruction qui le recouvre ${ }^{9}$. Dans ce deuxième temps, l'hallucination est une tentative de guérison (Freud, 1924 a, 285) en ce qu'elle vise à re-construire une certaine continuité avec la réalité externe. Elle « comporte le caractère de la réparation », visant à « compenser la perte de la réalité » (Freud, 1924 b, 300). Les reconstructions hallucinatoires recouvriraient ainsi l'effet du temps négatif de l'hallucination (Freud, 1924 a, 285). Comme le dit Freud « dans le tableau clinique de la psychose les manifestations du processus pathogène sont souvent recouvertes par celles d'une tentative de guérison ou de reconstruction » (Freud, 1924 a, 285). Les hallucinations sont alors comme Freud le soutient dans Névrose et psychose (1924a), des caches, des pièces « qu'on colle là où initialement s'était produite une faille dans la relation du moi au monde extérieur » (1924 a, 285). L'hallucination cache et indique tout à la fois, en négatif, un trou, une béance.

Par exemple, le fils d'une patiente, avec lequel elle avait une relation très fusionnelle, décède brutalement. Elle se met à le voir (l'halluciner) auprès d'elle. La situation intolérable et sa représentation (la pensée de la mort du fils) ont ainsi été rejetées et remplacées par une présence perceptive. Mais après un retournement ou l'absence est inversée en présence.

\section{$2^{\circ}$ La mise au premier plan de la représentation rejetée : le retour du rejeté}

Freud, et Lacan après lui, ont pensé l'hallucination comme un retour sous forme perceptive de la représentation rejetée (ou de la représentation potentielle rejetée). 
Pour Freud, ce qui a été aboli au dedans (ou rejeté) reviendrait du dehors sous forme perceptive (Freud, 1911,315) ${ }^{\circ}$; pour Lacan, ce qui a été forclos [c'est-à-dire rejeté] du symbolique reviendrait dans le réel (c'est-à-dire sous forme de perception) (Lacan, 1955-56,215). C'est ce qui amène Freud à parler du « noyau de vérité » des hallucinations : un sens, une signification " potentielle » y est enclose, même si elle n' a pu se développer pour devenir une pensée à cause du processus de rejet.

Nous pensons, après S. Freud, que l'hallucination se constitue à partir de ce qui a été rejeté et qui fait retour". C'est ce que nous proposons de nommer le « retour du rejeté $»^{12}$. Dans cette perspective, c' est à partir de la représentation potentielle rejetée que l'hallucination est construite. L'hallucination apparaît ainsi comme une formation de substitut (Freud, 1915) : elle se substitue à une représentation, à une pensée qui n'a pu advenir. L'hallucination est la perception d'un impensé.

\section{Le travail de I'hallucination}

\section{$1^{\circ}$ Pas un simple retour}

Je soutiens que l'hallucination n'est pas un simple retour, sous forme perceptive de la représentation rejetée (Freud, 1894), ou d'un signifiant forclos du symbolique (Lacan, 1955-56), mais le produit de transformations portant sur la représentation potentielle (intolérable) rejetée : c'est ce que je nomme le travail de l'hallucination.

\section{$2^{\circ}$ Notion de travail de I'hallucination}

Je propose d'appeler « travail de l'hallucination » (Gimenez, 1994) l'ensemble des mécanismes de transformation qui portent sur la représentation potentielle rejetée pour la transformer en hallucination. Ce travail s'effectue à partir de traces, laissée dans la psyché par F expérience intolérable. Il s'agit de ce que Freud nomme les représentations de choses (représentation-chose de J. Laplanche). J'utilise le terme de travail (de l'hallucination) au sens où Freud l'entend dans l'expression « travail délirant » (Freud, 1911, 315), quand le patient rebâtit l'univers de telle sorte qu'il puisse à nouveau y vivre ${ }^{13}$.

Dans cette perspective, la nouvelle réalité hallucinatoire se constituerait par transformations à partir de la représentation potentielle
10 « II n'était pas juste de dire que le sentiment réprimé au dedans fût projeté au dehors ; on devrait plutôt dire, nous le voyons à présent, que ce qui a été aboli au dedans revient du dehors. L'investigation approfondie du processus de la projection, que nous avons remise à une autre fois, nous apportera sur ce point des certitudes qui nous manquent encore » (Freud, 1911,315). 11 Comme le dit S. Freud : "Vraisemblable ment, dans la psychose, le fragment de réalité repoussé revient sans cesse forcer l'ouverture vers la vie psychique (Freud, 1924b, 302). Ainsi, la représentation rejetée fait ainsi retour dans l'hallucination, sous forme perceptive. 12 En 1896, dans les Nouvelles remarques, Freud qui n'a pas encore élaboré le concept de rejet, le dit avec le terme de refoulement: « J'avais donc appris que ces hallucinations n'étaient rien d'autre que des fragments des expériences infantiles refoulées, des symptômes du retour du refoulé ") (Freud, 1896, 78). 13 Précisons que le travail de l'hallucination, contrairement au travail du rêve, ne consiste pas à camoufler une représentation intolérable. II ne porte pas sur un contenu latent pour $\mathrm{k}$ 
(suite note 13)

transformer en contenu

manifeste et ainsi passer

la censure. II correspond

à l'ensemble des

transformations

effectuées sur la

représentation poten-

tielle, qui n'a pu se

construire, se constituer

en pensée. Ce travail

sert à la fois à se

protéger contre une

représentation intoléra-

ble et à construire une

forme qui constitue un

premier contenant (apte

à contenir une expé-

rience intolérable,

traumatique), même si

cette tentative ne

parvient pas à son

terme.

14 Freud évoque à

nouveau 26 ans plus

tard I'hallucination

comme « ce qui

s'impose [...] à la

conscience, mais

probablement de façon

déformée » (Freud,

1937,279).

15 A propos du rapport

de « L'homme aux

loups » à la « castra-

tion » lors de son

hallucination, Freud dit

qu'il l'a rejetée, l'a

reconnue, et enfin qu'il

commençait à la

reconnaître à l'époque

de son hallucination,

celle-ci ayant été une

aide dans cette

élaboration. II est

important de souligner

que l'hallucination peut

être ainsi pensée

comme un temps de

passage d'un mode de

fonctionnement à un

autre plus élaboré.

« Nous pouvons par

suite admettre que cette rejetée et de l'affect qui lui était associé (la tension produite par la réalité intolérable). Ce travail rend possible une figuration plus tolérable de la situation à laquelle le sujet est confronté. Dans cette perspective, on peut penser l'hallucination persécutive ou angoissante - par analogie avec le cauchemar - comme une hallucination qui aurait insuffisamment transformé la représentation potentielle de la situation intolérable et insuffisamment contenu sa charge d'affect (Gimenez, 1994). Précisons que le travail de l'hallucination, contrairement au travail du rêve, ne consiste pas à camoufler une représentation intolérable. 11 ne porte pas sur un contenu latent pour le transformer en contenu manifeste et ainsi passer la censure. Il correspond à l'ensemble des transformations effectuées sur la représentation potentielle, qui n' a pu se construire, se constituer en pensée. Ce travail sert à la fois à se protéger contre une représentation intolérable et à construire une forme qui constitue un premier contenant (apte à contenir une expérience intolérable, traumatique), même si cette tentative ne parvient pas à son terme (avorte). Il s'agit d'un dépôt instable (le déposé). L'idée de transformation ou de déformation précédant le retour n'est pas étranger à la démarche de Freud, même s'il ne le théorise pas. Il l'explicite clairement en 1911 dans son texte sur le Président Schreber : « Une perception interne [c'est-à-dire une représentation] est réprimée et, en ses lieux et place, son contenu, après avoir subi une certaine déformation, parvient à la conscience sous forme de perception venant de l'extérieur » (Freud, 1911, 311) ${ }^{14}$.

La notion de " travail de l'hallucination » ne constitue pas uniquement une défense mais permet également figurer de l'infigurable, à représenter de l'irreprésentable. Il pallie, en s'y substituant, le travail de la mise en pensée rendu impossible par le processus de rejet (Gimenez, 1994,1997). Il est un mouvement de symbolisation non parvenu à son terme, en cela nous $\mathrm{F}$ appelons « mise en représentation pré-symbolique $»^{15}$. La construction hallucinatoire serait une figuration, une mise en image ou " mise en forme " perceptive. Nous entendons le terme de «forme » dans le sens où $\mathrm{D}$. Anzieu le définit, c'est-à-dire « au sens premier du terme : « un ensemble des contours d'un objet, résultat de F organisation de ses parties, c'est-à-dire configuration, figure » (Anzieu, 1987,1).

Les formes hallucinatoires se constituent à partir de « représentations-choses », inscriptions d'expériences sensori-motrices ou sensoriaffectives non symbolisées. Ces représentations-choses, réinvesties et réactivées dans un mouvement régressif, sont souvent traduites par des sensations ou impressions corporelles : en cela elles renvoient au 
pictogramme (Aulagnier, 1975) et au signifiant formel (Anzieu, 1987). Nous soutenons que la mise en représentation présymbolique s'effectue à partir de traces, laissée dans la psyché par l'expérience intolérable. C'est ici le «magasin des accessoires » au sens freudien où sera puisé le matériel pour créer les hallucinations.

Je soutiens que ce travail de l'hallucination s'effectue à partir de traces, laissée dans la psyché par l'expérience intolérable. C'est ici le « magasin des accessoires » au sens freudien ou sera puisé le matériel pour créer les hallucinations. J'ai insisté sur la concrétude et la dimension corporéisée de ces traces (renvoyant au langage d'organe dont parle Freud). Quelle que soit la terminologie retenue pour nommer ces inscriptions (représentation de chose, signifiant formel, pictogramme, etc.), on peut s'accorder pour dire qu'il s'agit de représentation non symboliques. Si l'on accepte de s'inscrire dans une perspective évolutive, - et résolument optimiste - on les appellera représentation pré-symboliques.

Le terme freudien qui m'a paru le plus adéquat pour nommer ces traces, appartenant à la catégorie des représentations de choses, est le terme de trace motrice, qui trouve son prolongement dans la notion de « signifiant formel » conceptualisée par D. Anzieu. Trace inconsciente, traduisant une impression corporelle renvoyant aux enveloppes (par ex. « le cerveau se vide »), à des changements d'état du corps (un bras s'allonge, une oreille se détache de mon corps et se déplie).

\section{$3^{\circ}$ Les mécanismes du travail de l'hallucination}

Parmi ces mécanismes du travail de l'hallucination (Gimenez, 2000a ; Pedinielli, Gimenez, 2002), citons :

La démétaphorisation : mécanisme actif par lequel une expression ou une représentation perd sa dimension métaphorique. La démétaphorisation rejoint la notion de mise en équation symbolique dont parle M. Klein et $\mathrm{H}$. Segal et la mise en équivalence du mot avec la chose décrite par Freud. Elle aboutit à un mode de fonctionnement selon la pensée concrète. Elle amène également des scénarios très corporéisés : on peut ainsi considérer l'hallucination comme un langage d'organe, ce que Freud soutenait du délire. La descénarisation : est le mécanisme par lequel un scénario intolérable est transformé en un préscénario comme une représentation de mouvement (ou de transformation) (ce que D. Anzieu a nommé signifiant formel) ou en une représentation de sensation, c'est-à-dire un pictogramme tel que l'a conceptualisé Aulagnier. La régression formelle est le mécanisme (suite note 15) hallucination eut lieu à l'époque où il se décida à reconnaître la réalité de la castration ; peut-être marqua-t-elle justement cette démarche » (Freud, 1914, 390). 
16 Ce cas a été en partie présenté dans le texte « Le travail de I'hallucination » (Gimenez, 2000b). 17 II s'agit en fait d'une construction délirante fondée sur la naissance de la petite sœur quelques années auparavant ; Jacques pense que sa mère lui a préféré son frère après le départ à l'armée : « Après mon départ, elle l'a préféré, il était plus beau que moi "... La décompensation de Jacques s'inscrit ainsi dans un contexte où il est confronté à trois expériences émotionnelles intolérables pour lui : la séparation d'avec la cellule familiale (le départ à l'armée), la rivalité avec son frère et la naissance d'une petite sœur. par lequel une pensée potentielle est transformée en image (figuration) : au lieu d'être en contact avec une pensée intolérable, le sujet est en contact avec une image qui la figure. Par le mécanisme de projection cette image est appréhendée comme venant du dehors (ce qui la différencie d'une image mentale). La projection sous-tend le phénomène xénopathique des hallucinations. La dimension corporéisée de l'hallucination nous permet de parler ici d'excorporation. Par la mise en suspens du jugement de réalité, le sujet appréhende cette image venant du dehors comme une perception. Enfin, par la mise en suspens du jugement assertif ou d'existence, le sujet appréhende ce qu'il voit comme quelque chose qui existe.

\section{$4^{\circ}$ Le travail de l'interprétation}

Le travail clinique, thérapeutique, analytique, de l'interprétation vise à effectuer le travail inverse et permettre que la pensée rejetée puisse se construire, et que l'expérience puisse se symboliser, à l'intérieur d'une relation intersubjective (avec le clinicien et avec une équipe de soin). Il s'agit de la métaphorisation (ou re-métaphorisation), la scénarisation (ou re-scénarisation), la ré-introjection du projeté, la remise en place du jugement de réalité et du jugement d'existence pour cette expérience.

\section{Le travail de I'hallucination et son inter- prétation}

Je vais maintenant décrire quelques-uns de ces mécanismes du travail de l'hallucination, et leur élaboration possible (en particulier la démétaphorisation et la descénarisation), à partir de séquences cliniques issues de la thérapie de Jacques.

\section{$1^{\circ}$ Présentation de Jacques}

Jacques est un jeune homme de dix-huit ans, de stature athlétique, hospitalisé à la demande de ses parents très inquiets de ses passages à l'acte, de ses propos délirants et des voix qu'il dit entendre' ${ }^{6}$. Sa décompensation fait suite à son incorporation au service militaire. À l'armée, il s'est senti devenir une machine et a mis en acte ce que « la voix » lui demandait de faire : courir tout nu dans la caserne et frapper des gradés. Il est réformé et retourne au domicile parental ${ }^{17}$. Son père a aménagé un bureau dans la chambre du frère, qui a pris la chambre de Jacques. Cet événement est vécu comme un vol en faveur de son 
frère. Il pense que ses parents ne l'aiment plus, et qu'une petite sœur serait née pendant son absence, des amours incestueuses du frère avec la mère ${ }^{18}$. Les voix redoublent d'intensité et il frappe des membres de sa famille. Ceux-ci n'arrivent plus à assumer sa présence. Après un passage à l'acte sur son père, il est hospitalisé. Très réservé, il parle peu, ne se lie pas aux autres patients à l'hôpital et répond par « oui » ou par « non » aux soignants. Il reste de longs moments sans prononcer une parole. Ses silences sont émaillés d'attitudes d'écoute, de sourires (ou rires) discordants et de coups d'œil vers différents endroits de la pièce qu'il observe très attentivement.

\section{$2^{\circ}$ Les expériences d'éparpillement}

Dans une première période de la prise en charge, Jacques parle de façon très morcelée : il commence une phrase, en continue une autre, se met à rire, se lève, tend l'oreille. Son visage se crispe comme s'il était soudain inquiet. Il se rassied, regarde ses mains d'un air effrayé, les agite comme s'il ne les contrôlait pas. Quelquefois, il regarde, pétrifié ce qui se passe sur le mur ou dans un coin de la pièce, sans pouvoir verbaliser ce qu'il vit et prononce des sons inarticulés. Jacques est en proie à de nombreuses hallucinations pendant les entretiens. Il sent et voit ses mains se transformer, a l'impression que son corps devient une machine (il ressent des coups dans ses tympans), entend des voix, sent ses organes se disperser dans la pièce ${ }^{19}$.

\section{$3^{\circ}$ La démétaphorisation : la voix perçante de la mère et les " gros mots »}

Jacques éprouve également de façon auditive et cénesthésique des chocs dans son oreille droite. Il entend et sent ces chocs très violents lui percer les tympans. Jacques place souvent les mains sur ses oreilles. Il peut rester de longs moments à crier, la tête entre ses mains. Un jour, pour arrêter les voix, il reste devant un miroir, plusieurs heures, figé, en tenant un couteau au-dessus de son oreille. Il le nommera après coup le « couteau de survie ». Cette coupure de l'oreille apparait également dans ses hallucinations. Dès le premier mois de thérapie, Jacques parvient à parler, très tendu, des voix qui tapent dans ses oreilles et percent ses tympans. Lors d'un entretien, il dit : « II y a des voix qui tapent dans les tympans, qui percent $»^{20}$. Je fais le lien avec le dernier entretien et dis : «Vous disiez la dernière fois que votre mère a tapé dans les tympans ». « Oui, dit-il, elle a la voix perçante... elle a la voix aiguë, (« elle a la voix pointue »)... Je dois lui envoyer des claques ».
18 II s'agit en fait d'une construction délirante fondée sur la naissance de la petite sœur quelques années auparavant ; Jacques pense que sa mère lui $a$ préféré son frère après le départ à l'armée : «Après mon départ, elle l'a préféré, il était plus beau que moi »... La décompensation de Jacques s'inscrit dans un contexte où il est confronté à trois expériences émotionnelles intolérables pour lui : la séparation d'avec la cellule familiale (le départ à l'armée), la rivalité avec son frère et la naissance d'une petite sœur.

19 L'évocation de ses hallucinations semble provoquer en lui des montées de tension importantes souvent repérables au niveau contre-transférentiel. On peut alors ressentir une tension insupportable, presque palpable, comme le décrit $W$. $R$. Bion (1962) au sujet des éléments bêta, équivalents à une « chose en soi ».

20 Je suis frappé que Jacques utilise les mêmes expressions pour parler de la voix et pour parler de sa mère, mais de façon non métaphorique. 
Je reprends : « Votre mère parle avec sa voix pointue, aiguë, elle tape sur vos tympans, elle crève vos tympans de sa voix perçante et vous tapez sur sa figure ». Il dit : « Oui, comme ça... elle l'avait bien mérité ». Et Jacques précisera qu'il tape sur les oreilles de ses interlocuteurs : signifiant ainsi que le passage à l'acte figurait, en miroir, le même scénario que l'hallucination : " On tape sur une oreille ». Jacques parlera très souvent de ces coups dans les tympans comme d'une expérience très violente, intrusive, le plus souvent associée à la mère qui, dit-il, lui « casse les oreilles », comme il dit.

Il est troublant de noter une réflexion de soignants qui ont reçu la mère : quand elle commence à évoquer son fils Jacques, sa voix se « casse », devient aiguë et stridente, perçante, discordante. Dans ses hallucinations, Jacques reprend ainsi de façon non métaphorique (en le démétaphorisant) un aspect perceptif du ton de la voix de la mère : sa « voix cassante », expression à entendre au pied de la lettre ; comme une équation symbolique (Segal, 1957). Jacques parle également de sa douleur de ne pas recevoir des mots qui ne viennent pas. Ainsi, son oreille est-elle « en attente de mots d'affriction » « qui ne peuvent entrer » dans son oreille parce qu'ils sont trop gros, dit-il. « Ma mère dit des gros mots, des mots trop grands », précise-il. Jacques associera trois ans plus tard ces phénomènes à l'impossibilité à s'écouter et à se parler dans la famille. Ce néologisme («affriction ») apparaît dans ses associations comme une condensation entre l'affection et la friction, entre un sentiment affectueux ( affection ») et une expérience hostile (« friction »). Et peut-être également l'affliction. Le matériel clinique indiquera que ce néologisme traduit la relation de Jacques à sa mère qu'il vit comme à la fois intrusive (choc, friction, voix perçante) et inaffective (affection en attente).

Les hallucinations de choc dans les tympans semblent figurer des modalités relationnelles à la mère qui n'ont pu être mises en pensée : des mots attendus qui ne viennent pas, d'autres qui sont reçus comme des projectiles qui attaquent et percent (la voix perçante). Ceci dans une alternative : ou ne pas s'entendre (oreille gauche), ou être intruse (oreille droite), dans un clivage vertical de l'image du corps.

La relation à la mère, appréhendée par Jacques, dans ce temps de la thérapie, comme intrusive et inaffective, revient sous formes d'hallucinations auditives et cénesthésiques de « gros mots »,des « mots trop grands » (voix perçante, voix pointue), tels des projectiles qui tapent sur ses oreilles et percent ses tympans. L'hallucination apparaît ici comme le résultat d'une transformation démétaphorisante d'un aspect de la relation non pensée à l'objet. 


\section{$4^{\circ}$ La descénarisation}

Je fais l'hypothèse que la descénarisation a transformé le scénario de base intolérable [que l'on peut traduire sous la forme d'une phrase : d'un sujet, d'un verbe et d'un complément] « on m'intruse » ou « on me perce les tympans » d'abord en signifiant formel « un tympan se perce », puis en pictogramme. Nous n'avons plus alors de scénario ni de pré-scénario, ni même de verbe, mais seulement une sensation (de surface), résultat de l'action des verbes : percer, frapper ${ }^{21}$ que l'on retrouve dans les hallucinations : la sensation d'un tympan qui se perce et se déchire.

\section{$5^{\circ}$ La re-scénarisation}

Jacques évoque son hallucination comme une expérience sensorielle : il éprouve dans son corps une sensation : celle d'un choc violent sur son tympan. Tout son être est centré sur la sensation violente, traumatique, produit de la rencontre entre son tympan et un objet pointu, froid, tel un clou qui vient le percer, le déchirer. Nous sommes prêt de la définition du pictogramme dont parle Piera Aulagnier, résultat du contact entre une partie du corps et un objet externe, représentationsensation en deçà des mots, comme celle de l'alpiniste qui glisse et se raccroche dans un ultime geste à une aspérité du rocher sur une paroi glissante : sa vie dépend de cette sensation sur laquelle tout son être se centre. Ici, le pictogramme apparaît bien comme l'effet du contact entre une partie du corps du sujet investie (ici l'oreille) et sa zone complémentaire (la bouche de la mère et les sons intrusifs qui en sortent). Mais quand Jacques évoque cette sensation corporelle, il la transforme. Dans renonciation à un autre que lui-même, il transforme la sensation hallucinatoire en un énoncé : « un tympan se perce ». Il s'agit alors non plus d'une sensation mais d'une représentation de mouvement ou de transformation, ce que Freud appelle une image motrice. C'est aussi ce que Anzieu nomme un « signifiant formel» : un pré-scénario composé d'un sujet identifié à une partie du corps (le tympan) et d'un verbe réflexif (se perce) : " un tympan se perce ». Nous repérons ici la dimension "représentation de mouvement», renvoyant à ce que Freud avait commencé à conceptualiser sous le terme d'image motrice (1895 a).

Et c'est dans la dynamique de la relation inter-subjective, par le travail de liaison, que ce pré-scénario (re)deviendra progressivement un scénario : « la voix, tout comme ma mère, m'intruse, me perce, me crève les tympans ». Je fais l'hypothèse que la re-scénarisation dans la
21 La notion de pictogramme de met en évidence la dimension de « sensation de surface » dans la coalescence à « l'objetzone complémentaire ». 
thérapie effectue le travail inverse de la de-scénarisation du travail de l'hallucination. C' est pour nous, le travail de la rencontre avec $\mathrm{F}$ autre, dans un cadre psychothérapique, qui permet à l'hallucination de se (re)scénariser et se (re)métaphoriser. Voici une courte séquence clinique où l'on voit à $\mathrm{F}$ œuvre cette resocialisation et remétaphorisation.

\section{$6^{\circ}$ La métaphorisation symbolisante dans le travail clinique}

Au cours d'un entretien, Jacques semble soudain affolé ; il tourne brusquement les yeux vers le sol, inquiet. Je regarde dans la même direction et je lui dis qu'il semble très préoccupé de ce qui se passe par terre. "Elle est tombée », reprend-il tout doucement (il indique l'oreille droite). Il explore activement l'espace qui se trouve devant lui, à sa gauche, à sa droite, puis, de son regard, fixe le coin du bureau, comme s'il surveillait un objet qui se trouverait là. Mes yeux suivent le déplacement des siens. Je me surprends à me laisser aller à rêver ce qu'il halluciné peut-être : une oreille là, rendant ainsi co-présentes deux représentations, deux figurations d'une expérience ayant des statuts différents, la rêverie et l'hallucination. Je l'accompagne de mon regard. Je verbalise alors, également à voix basse : «Vous regardez le coin du bureau fixement comme si vous aviez trou vé ce que vous cherchiez ».

« Elle est là », continue-t-il, en me regardant, indiquant du doigt un coin du bureau. Invité à mettre en mots ce qu' il ressent, Jacques précise après un silence qu'il voit son oreille comme il me voit, " trouble ». J'entends sa phrase dans une dimension transférentielle : il me voit de la même façon que l'oreille hallucinée, trouble. Il a perdu une oreille et en retrouve une autre, ouverte, dépliée, plus grande, et trouble.

Peut-être commence-t-il à m'investir comme une oreille qui peut l'écouter. Je reprends : « Vous avez senti que votre oreille est tombée, vous l'avez cherchée du regard, et repérée au coin du bureau, plus grande, dépliée et trouble, comme moi ». Jacques reste encore quelques instants à la surveiller puis semble plus détendu.

Trois entretiens plus tard, Jacques a l'air encore préoccupé par son oreille. Il dit : « Je l'ai touchée l'autre jour... je crois que ça m'a fait mal [... ] ça m'a fait mal entre moi... ». Je reprends : « Entre vous ?». « Oui, ma famille » précise-t-il. Je tente de reformuler la ligne associative avec les mots de Jacques puis avec les miens, c'est-à-dire en insérant des écarts dans la fusion et l'indifférenciation entre lui et sa famille : « Votre oreille est tombée, dépliée, vous F avez touchée, et ça 
vous a fait mal entre vous : votre famille; entre vous et votre famille ». Il semble que l'expression " entre moi » concernant la perte de l'oreille, traduise bien la relation encore indifférenciée et fusionnelle entre Jacques et sa famille, qui s'élabore progressivement.

Nous avons repéré le mouvement de transférentialisation des hallucinations : l'oreille tombe et Jacques la retrouve sur le coin de mon bureau, trouble comme moi. Mais l'oreille retrouvée présente quelques différences, quelques écarts avec l'oreille perdue : elle porte la trace du thérapeute : elle est plus grande, et dépliée (prête à écouter). Alors qu'elle se transforme dans la dynamique intersubjective (transférentialisation), l'hallucination se scénarise et permet que se rejoue, dans la relation clinique, ce qui restait enclos, gelé dans le scénario hallucinatoire (je suis l'oreille qui l'écoute).

Dans la dynamique transférentielle, l'hallucination évolue de façon élaborative. Cette évolution vers la pensée et la représentation symbolisée laisse progressivement apparaître des places dans le scénario (ou pré-scénario) hallucinatoire, signant l'émergence d'un espace pour la séparation et la différenciation (« entre moi », « moi et ma famille »). Jacques remarque, au bout de quelques mois, que les voix sont moins fortes au cours des entretiens. Elles parlent moins fort et moins fréquemment (diminution de l'intensité et de la quantité). Mais si le cadre des entretiens a une fonction pare-excitative, elle n'est encore que de courte durée : quand quelqu'un l'écoute, quand une oreille est disponible pour lui, ses oreilles sont moins remplies par les voix.

\section{Conclusion}

Les hallucinations de Jacques, indiquent, de façon transformée, les représentations potentielles intolérables contre lesquelles le patient s'est défendu, en les rejetant. Elles en sont des figurations substitutives pré-symboliques. Revient dans l'hallucination, appréhendé de façon perceptive, ce qui a été rejeté, mais après un travail de transformation que nous avons nommé " le travail de l'hallucination ». L'étude d'hallucinations auditives non verbales de Jacques (les chocs dans les tympans) nous a permis d'en étudier deux mécanismes de ce travail de l'hallucination : la démétaphorisation et la descénarisation.

L'hallucination nous est ainsi apparue comme une figuration démétaphorisée d'un scénario qui n'a pu se mettre en pensée. Il s'agit d'une mise en équation symbolique sensorielle de vécus affectifs non 
encore intégrés, non digérés comme le dit Bion. Les représentations (potentielles) rejetées (non advenues) et restées impensées (sans penseur dirait Bion) se concrétisent (après quelques transformations) de façon sensorielle, auditive. L'hallucination, comme formation de substitut apparaît en lieu et place d'une pensée qui n'a pu advenir, ou parvenir à son terme : une pensée rejetée. Ainsi, le vécu d'intrusion et celui d'attente affective sont représentés de façon démétaphorisée par des hallucinations de voix perçante (un tympan se perce, une oreille se casse).

L'hallucination nous est également apparue comme le résultat d'une déscénarisation de la représentation potentielle (représentation intolérable rejetée). Le scénario intolérable qui ne pouvait pas être pensé est ainsi transformé en un pré-scénario comme un signifiant formel (une oreille se crève, un tympan se perce, une enveloppe protectrice se détruit, disparaît), et même en un pictogramme (ou il est réduit en une sensation : comme celle du choc sur les tympans). Dans la relation clinique, le mouvement de transférentialisation des hallucinations (l'oreille qui tombe et que Jacques retrouve sur le coin de mon bureau : trouble, comme moi) permet que soit relancé le travail de la remétaphorisation symbolisante et de la re-scénarisation. Nous pouvons, ainsi, accompagner les patients, comme Jacques, de la perception de l'impensé (leurs hallucinations) à la pensée de ce qui est resté trop longtemps une expérience traumatique en attente d'un espace où il pourrait un jour être reçu et transformé pour devenir intégrable? C' est en effet pour autant qu'une forme hallucinatoire soit construite, et que cette forme soit adressée à un autre que soi, que pourra être relancé le travail de symbolisation.

\section{Bibliographie}

ANZIEU.D., 1987. Les signifiants formels elle Moi-peau. In ANZIEUD..HOUZEL D., et coll. Les enveloppes psychiques. Paris : Dunod, 1-22.

AULAGNIER, P., 1975. La violence de l'interprétation. Dupictogramme à l'énoncé. Paris : Presses Universitaires de France.

BION, W.R., 1962. Aux sources de l'expérience. Paris : Presses Universitaires de France, 1979.

BOTELLA, C., BOTELLA, S., 1989. La problématique de la régression formelle de la pensée et de l'hallucinatoire. Revue Française de Psychanalyse, Colloque S.P.P. Unesco du 14 et 15 janvier 1989, 63-90.

FREUD, S., 1894. Les psychonévroses de défense (Essai d'une théorie psychologique de l'hystérie acquise de nombreuses phobies et obsessions et de certaines psycho ses hallucinatoires). In Névrose, psychose et perversion. Paris : Presses Universi taires de France, 1973, 1-14. 
1895. L'esquisse d'une psychologie scientifique. In La naissance de la psychana lyse. Paris : Presses Universitaires de France, 1956, 313-396.

1896. Nouvelles remarques sur les psychonévroses de défense. In Névrose, psychose et perversion. Paris : Presses Universitaires de France, 1973,61-81.

1911. Remarques psychanalytiques suri' autobiographie d'un cas de paranoïa (Le président Schreber). In Cinq psychanalyses. Paris : Presses Universitaires de France, 1970,263-321.

1914 c. Extrait de l'histoire d'une névrose infantile (L'homme aux loups). In Cinq psychanalyses. Paris : Presses Universitaires de France, 1970, 325-420.

1915. L'inconscient. In Métapsychologie. Paris : Gallimard, 1940, 65-123. 1924 a. Névrose et psychose. In Névrose, psychose et perversion. Paris : Presses Universitaires de France, 1973,283-286.

1924 b. La perte de la réalité dans la névrose et la psychose. In Névrose, psychose et perversion. Paris : Presses Universitaires de France, 1973,299-303.

Gimenez, G., 1994b. Entre chaos et pensée, l'hallucination, un contenant présymbolique. In : D. Anzieu (Ed.). L'activité de pensée, émergences et troubles (pp. 145-156). Paris : Dunod.

1997. Des Voix àlaparole. In Green A., BotellaS.,BotellaC.,Boubli M., Gimenez G., Aulagnier P., Fonagy J., Pinol-Douriez M., (Eds.). Pulsions, représentations, langage. Théorie et clinique psychanalytiques (pp. 93-133). Delachaux et Nieslé. 2000a. Le travail de l'hallucination. Cliniques Méditerranéennes, 62, 149-167. 2000b. Clinique de l'hallucinationpsychotique. Paris, Dunod. Ouvrage traduit en italien : Gimenez, G. (2002). Clinica dell'allucinazione Psicotica. Roma, Borla. GREEN, A., 1977. L'hallucination négative. Notes pour un addendum à un traité des hallucinations. Hommage à Henri Ey. L'évolution psychiatrique, XLII, fasc. III/ 2,645-656.

1983. Le langage dans la psychanalyse. In : A.. GREEN, R. DIATKINE, E. JABES, M. FAIN, I. FONAGY, Langages. Ilième rencontre psychanalytiques d'Aix-en-Provence, les Belles Lettres, 19-250. LACAN, J., 1953-1954. Les écrits techniques de Freud. Le Séminaire, Livre 1. Paris :

Seuil, 1975.

1955-1956. Les Psychoses, Le séminaire, Livre III. Paris : Seuil, 1981.

LAPLANCHE, I., PONTALIS, J.B., 1967. Vocabulaire de la psychanalyse. Paris : Presses Universitaires de France. NASIO, J.D., 1987. Les yeux de Lame, le concept d'objet a dans la théorie de J.

Lacan. Paris : Aubier.

PEDINIELLI, I.L., GIMENEZ, G., 2002. Les psychoses de l'adulte. Collection 128. Paris, Nathan.

ROSOLATO, G., 1964. Essais sur le symbolique. Paris : Gallimard, 1969. 
Résumé L'hallucination, dans une perspective psychanalytique peut être considérée comme la perception d'une expérience qui n'a pas pu se penser ou se symboliser, et dont le modèle serait le trauma. L'hallucination serait une figuration, appréhendée comme perception d'un impensé. Freud, et Lacan après lui, pense ainsi l'hallucination comme le retour, appréhendé sous forme perceptive, de représentations rejetées (ou forcloses). L'hallucination serait ainsi une figuration, appréhendée comme perception, d'un impensé, d'une expérience qui n'a pas pu se penser ou se symboliser. L'auteur complète l'hypothèse freudienne du retour sous forme de perception de ce qui n'a pu être représenté : ce retour se ferait seulement après des transformations. L'ensemble de ces transformations constitue pour l'auteur le Ç travail È de l'hallucination. Dans ce texte, sont décrits quelques-uns des mécanismes du travail de l'hallucination, ainsi que leur interprétation possible, à partir de séquences cliniques de la thérapie d'un jeune patient schizophrène.

Mots-clés Hallucination, délire, rejet, symbolisation, figuration, descénarisation, psychose, schizophrénie.

Summary Hallucination, in psychoanaly tic psychopathological perspective could be considered as perception of an expérience mat has not been thought or symbolized, and whose model could be thé trauma. Hallucination would be a figuration, apprehended as perception, of an non-thought. Hallucinations are considered by Freud and Lacan like thé return, and thé concrétisation under a perceptive form, of rejected représentations (or foreclosed).

Thus, hallucinations would be a figuration of a non symbolised expérience, apprehended then as perception. Thé author complète Freud hy pothesis of thé return in thé form of perception of what has not been represented : this return would take place only after transformations. Totality of thèse transformations constitutes for authors thé « work » of hallucination.

In this text, are described some of thé mechanisms of thé work of hallucination, and their possible interprétation, from clinic séquences from thé therapy of a young schizophrénie patient.

Keywords Hallucination, delirium, reject, symbolization, figuration, démétaphorisation, psychosis, schizophrenia. 